\title{
Measurement of ion species ratio in the plasma source ion implantation process
}

\author{
B. Y. Tang, R. P. Fetherston, M. Shamim, R. A. Breun, A. Chen, and J. R. Conrad \\ Department of Nuclear Engineering and Engineering Physics, University of Wisconsin-Madison, \\ 1500 Johnson Drive, Madison, Wisconsin 53706
}

(Received 22 October 1992; accepted for publication 12 January 1993)

\begin{abstract}
Ion species and their ratios in nitrogen, oxygen, and argon plasmas in the plasma source ion implantation process have been determined with a simple and low-cost measurement system. The measured ion species ratio in the nitrogen plasma was used as an input parameter for the computer simulation code transport and mixing from ion irradiation to predict the atomic composition-depth profile. Comparison between the code results and data derived from Auger analysis for a nitrogen-implanted Ti-6Al-4V alloy showed good agreement. In this article, the design, performance, and possible future improvements regarding the resolution of this measurement system will be discussed.
\end{abstract}

\section{INTRODUCTION}

Plasma source ion implantation (PSII) is a costeffective technique for surface modification of nonplanar targets. ${ }^{1,2}$ In the PSII process, targets to be implanted are placed directly in a plasma source and then pulse biased to a high negative potential. A plasma sheath forms around the target and accelerated ions bombard the target normal to the surface. ${ }^{3}$ Unlike the conventional beam line ion implantation, the PSII is a nonline-of-sight process. This makes it more cost effective for metallurgical applications, which usually involve nonplanar targets. The cost effectiveness stems from two factors: (1) target manipulation in the vacuum chamber is not necessary to achieve uniform implantation and (2) target masking, which may be required in beam line implantation to minimize target sputtering due to nonnormal beam, is not necessary. The effectiveness of the PSII process in improving the wear and corrosion resistance of engineering materials, industrial components, and orthopedic applications has been demonstrated. ${ }^{4,5}$ It has also been recently used in semiconductor processing. ${ }^{6}$

In PSII, two parameters that have strong influence on physical properties of implanted targets are the dose and energy of implanted ions. Accurate determination of ion species ratios is critical in predicting both the dose and energy of implanted ions. For example, if there are $25 \%$ $\mathrm{N}^{+}$and $75 \% \mathrm{~N}_{2}^{+}$in a nitrogen plasma, the average number of atoms produced per ion equals $1.75(0.25 \times 1+0.75$ $\times 2=1.75$ ). When the target is biased at $50 \mathrm{kV}$, after implantation, each implanted $\mathrm{N}^{+}$is converted into one nitrogen atom with $50 \mathrm{keV}$ energy and each implanted $\mathrm{N}_{2}^{+}$ is converted into two nitrogen atoms with $25 \mathrm{keV}$ energy. So, the knowledge of ion species and their ratios in the plasma is critical in predicting the implantation dose, atomic composition-depth profile, and eventually the effectiveness of the PSII process for a given application.

The ion species and their ratios in the PSII plasma depend on various process parameters, such as gas pressure, discharge current (or plasma density), and nature of the gas. The process by which ion species ratios change with these parameters is too complicated to be expressed as a mathematical function. Hence, the ion species and their ratios cannot be easily predicted theoretically and must be measured experimentally. Measurement of ion species ratios in plasmas has received some attention in recent years. $^{7-11}$ But, ion species ratios in the PSII plasmas and the change of the ion species ratios with the PSII process parameters have not been studied in detail.

The ion species and their ratios in nitrogen, oxygen, and argon plasmas in the PSII device have been determincd with a low-cost measurement system that differs from some conventional measurement systems. ${ }^{7,8}$ In this article, the construction of the system and some measured results are described. The measured ion species ratio in the nitrogen plasma was used as an input parameter for the computer simulation transport and mixing from ion irradiation (TAMIX) to predict the atomic composition-depth profile. A nitrogen-implanted flat Ti-6Al-4V sample was analyzed with the scanning Auger microprobe (SAM) to obtain experimental data of the atomic composition-depth profile. The comparison between the theoretical prediction and data derived from Auger analysis showed good agreement. Suggestions for future improvements regarding the resolution of the system are also discussed briefly.

\section{MEASUREMENT SYSTEM AND PRINCIPLE}

A schematic illustration of the measurement system is shown in Fig. 1. The measurement device consists of the following components: the low-energy sampling ion source, ${ }^{12}$ the $\mathbf{E} \times \mathbf{B}$ mass separator, ${ }^{13,14}$ the Faraday cup, and the pumping system.

\section{A. Low energy sampling ion source}

The measurement device is encapsulated in an aluminum cylinder $150 \mathrm{~mm}$ in diameter and $210 \mathrm{~mm}$ in length. The cylinder is connected to the PSII chamber wall and used as a part of the anode of the plasma source at ground potential. The gaseous discharge takes place between the anode and filaments of the plasma source. The plasma is produced in the space between the chamber wall and the 


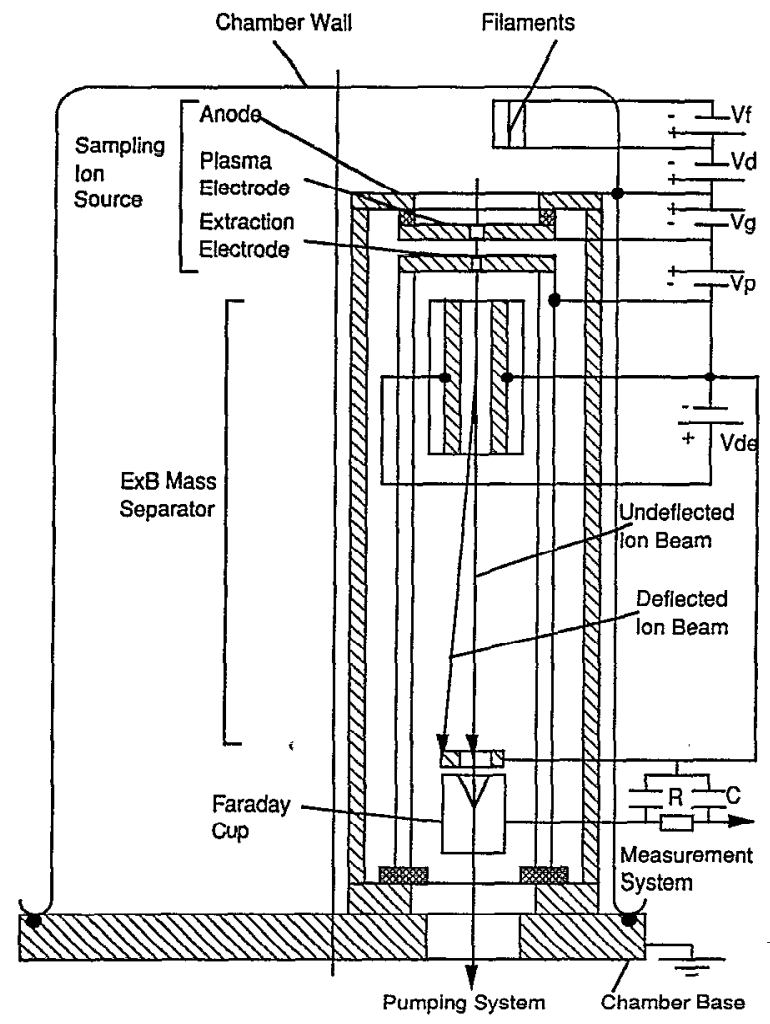

FIG. 1. Schematic illustration of the measurement system.

cylinder. An extraction system with two electrodes extracts a focused ion beam from the plasma. The extracted ion beam includes all the ion species that are present in the plasma.

The velocity of the extracted ions, $v$, depends on the acceleration voltage $V_{a},\left(V_{a}=V_{g}+V_{p}\right.$, where $V_{g}$ is bias voltage at the plasma electrode and $V_{p}$ is bias voltage at the extraction electrode). The velocity, $v$, can be expressed as

$$
v=\left(2 q V_{a} / m\right)^{1 / 2},
$$

where $q$ and $m$ are charge and mass of the ion, respectively.

\section{B. EXB mass separator}

In essence, the $\mathbf{E} \times \mathbf{B}$ mass separator is a velocity filter. When the extracted ion beam enters the $\mathbf{E} \times \mathbf{B}$ mass separator, ions are subjected to both magnetic and electric forces, simultaneously. If the two forces acting on the ion with charge $q$, mass $m$, and velocity $v$ balance each other, i.e.,

$$
q \mathbf{v} \times \mathbf{B}=q \mathbf{E} \text { or } v B=E,
$$

where $\mathbf{} \perp \mathbf{B}, \mathbf{B}$ is deflection magnetic field intensity produced by permanent magnets, and $\mathbf{E}$ is deflection electric field strength, the ion passes through the $\mathbf{E} \times \mathbf{B}$ mass separator directly and reaches the Faraday cup on the detecting plate without any deflection. The mass number $A_{0}$ of the undeflected ions can be deduced from Eqs. (1) and (2) as follows:

$$
A_{0}=1.9 \times 10^{-4} Z_{0} V_{a}(B / E)^{2},
$$

where $Z_{0}$ is the charge number of the undeflected ions, and $E=V / d$, where $V$ is deflection voltage and $d$ is distance between the deflection plates. From Eq. (3), it is obvious that if $V_{a}$ and $B$ are constants, the mass number $A_{0}$ of the undeflected ions can be considered to be a simple function of the deflection voltage.

The mass dispersion (or resolution) of the $\mathbf{E} \times \mathbf{B}$ mass separator can be described as a separated distance, $D_{m}$, in the radial direction at the entrance of the Faraday cup for the two ions with the same charge number, the same energy, and a mass number difference of $\Delta A=1$. The separated distance can be expressed using Eq. (4), i.e.,

$$
D_{m} \cong S L E / 4 A_{0} V_{a},
$$

where $L$ is the distance from the center of the deflection plates to the entrance of the Faraday cup and $S$ is the length of the deflection plates. $D_{m}$ is $4.6 \mathrm{~mm}$ for $\mathrm{N}^{+}$. Compared to the diameter $(2.97 \mathrm{~mm})$ of the entrance of the Faraday cup, the value of $D_{m}$ is big enough to separate $\mathrm{N}^{+}$ and $\mathrm{N}_{2}^{+}$.

As mentioned above, the mass number $A_{0}$ of the ion that is undeflected passing through the $\mathbf{E} \times \mathbf{B}$ mass separator depends on the ratio $(B / E)$, but from Eq. (4), it is clear that resolution depends on absolute values of $B$ and $E$. The resolution $D_{m}$ should be large enough to ensure that the two ions with mass number $A_{0}$ and $A_{0}+1$ (or $A_{0}-1$ ) will not overlap each other on the entrance of the Faraday cup.

\section{The Faraday cup}

The diameter of the entrance hole of the Faraday cup is $2.97 \mathrm{~mm}$. The distance from the middle of the deflection plates to the entrance of the Faraday cup is $100 \mathrm{~mm}$. The Faraday cup receives undeflected ions and sends the signal to a measurement system.

\section{Pumping system}

The dynamic gas pressure in the PSII chamber ranges from $1 \times 10^{-4}$ to $9 \times 10^{-4}$ Torr. In order to reduce an ionneutral particle (such as a residual gas molecule) interaction in the ion extraction region and the ion drift spaces, lower gas pressure must be maintained inside the measurement system. The current differential pumping between the PSII chamber and the inside of the measurement system is performed by means of the turbomolecular pumping system of the PSII device. As a result, gas pressure of less than $8 \times 10^{-5}$ Torr is consistently maintained inside the measurement system. This pressure is acceptable for nitrogen and oxygen plasmas because the charge exchange meanfree path ranges from 300 to $600 \mathrm{~cm}$ for $100-400 \mathrm{eV}$ ions, which leads to $3 \%$ to $6 \%$ ion loss before reaching the Faraday cup. For methane plasma, one needs lower pressure. A future plan for the modification of this device includes a new pumping system that would ensure a suffciently low pressure. The details of the modification and error estimation has been left to a future article. 


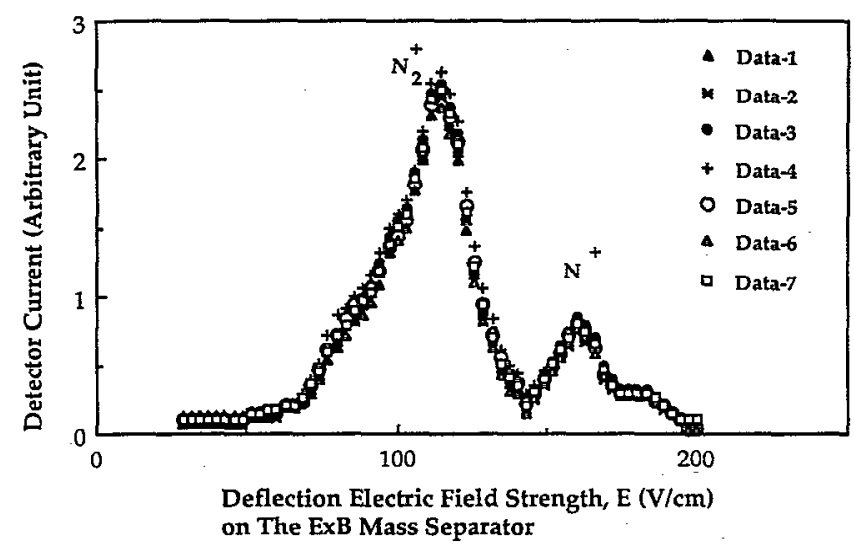

FIG. 2. Ion mass spectrum measured in a nitrogen plasma under the following conditions: $V_{d}=100 \mathrm{~V}, V_{\mathrm{g}}=-80 \mathrm{~V}, V_{a}=-410 \mathrm{~V}, P=1.5$ $\times 10^{-4}$ Torr, $I_{d}=60 \mathrm{~mA}, B=2150 \mathrm{G}$, and $E: 114.3 \mathrm{~V} / \mathrm{cm}$ for the peak of $75.7 \% \mathrm{~N}_{2}^{+}$and $160.0 \mathrm{~V} / \mathrm{cm}$ for the peak of $24.2 \% \mathrm{~N}^{+}$.

\section{ION SPECIES MEASUREMENT AND ANALYSIS}

The measurement of ion species and their ratios in nitrogen, oxygen, and argon plasmas were performed with the measurement system in the range of the PSII operating parameters. The gas pressure was varied from $8.0 \times 10^{-5}$ to $8.0 \times 10^{-4}$ Torr while the discharge current was varied from 20 to $80 \mathrm{~mA}$.

\section{A. Ion mass spectrum in nitrogen plasma}

Figure 2 shows the ion mass spectrum measured in a nitrogen plasma under the following conditions: $V_{d}=100$ $\mathrm{V}, V_{g}=-80 \mathrm{~V}, V_{a}=-410 \mathrm{~V}, P=1.5 \times 10^{-4}$ Torr, and $I_{d}=60 \mathrm{~mA}$. It may be determined from this spectrum and Eq. (3) that there are $\mathrm{N}^{+}$and $\mathrm{N}_{2}^{+}$peaks in the plasma with ratios of $\mathrm{N}^{+}=24.2 \%$ and $\mathrm{N}_{2}^{+}=75.7 \%$, where the deflection magnetic field intensity $B$ is $2150 \mathrm{G}$ and the deflection voltage is $80 \mathrm{~V}$ for the $\mathrm{N}_{2}^{+}$peak and $112 \mathrm{~V}$ for the $\mathrm{N}^{+}$peak, respectively.

Figure 3 shows the ion species ratios measured in a nitrogen plasma as a function of gas pressure $P$ under the following conditions: $V_{d}=120 \mathrm{~V}, V_{g}=-80 \mathrm{~V}, V_{a}=-410$

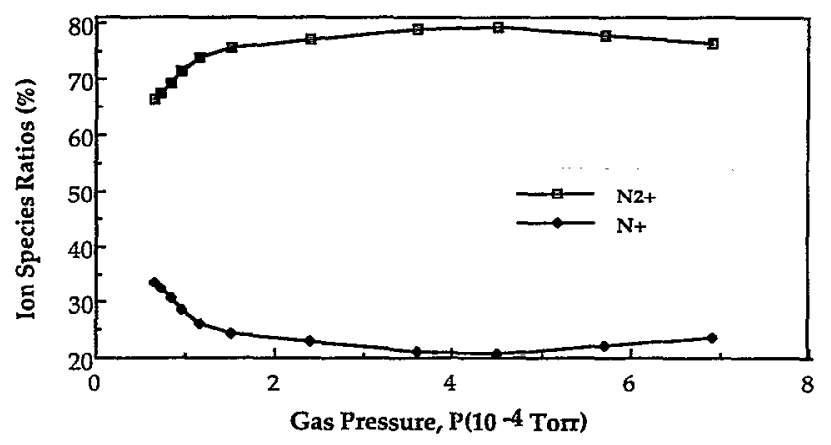

FIG. 3. Ion species ratios measured in nitrogen plasma as a function of gas pressure $P$ under the following conditions: $V_{d}=120 \mathrm{~V}, V_{g}=-80 \mathrm{~V}$, $V_{a}=-410 \mathrm{~V}$, and $I_{d}=30 \mathrm{~mA}$.

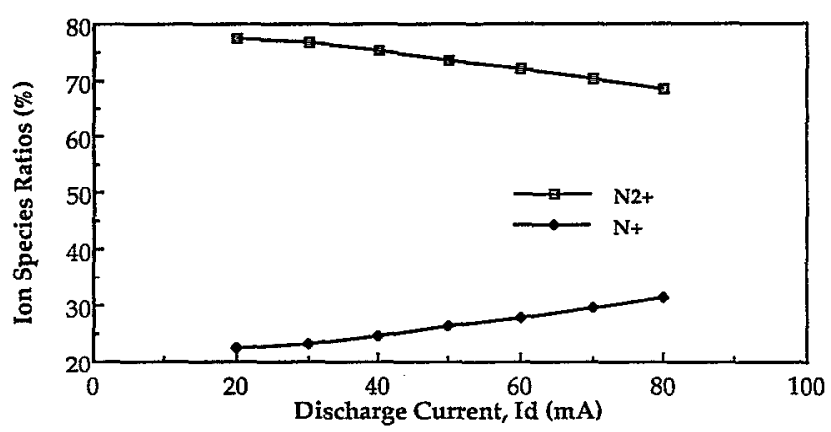

FIG. 4. Ion species ratios measured in a nitrogen plasma as a function of discharge current $I_{d}$ under the following conditions: $V_{d}=120 \mathrm{~V}, V_{8}$ $=-80 \mathrm{~V}, V_{a}=-410 \mathrm{~V}$, and $P=2.0 \times 10^{-4}$ Torr.

$\mathrm{V}$, and $I_{d}=30 \mathrm{~mA}$. It is noted that the $\mathrm{N}_{2}^{+}$percentage increases with gas pressure, reaches the maximum, and then decreases slowly with gas pressure.

We also measured the total extracted ion beam current as a function of gas pressure under the following conditions: $V_{d}=100 \mathrm{~V}, V_{g}=-80 \mathrm{~V}, V_{a}=-380 \mathrm{~V}$, and $I_{d}=60$ $\mathrm{mA}$. The measured result shows that the total extracted ion beam current is the maximum at approximately 4.5 $\times 10^{-4}$ Torr.

At lower gas pressures, the mean electron temperature is higher than at higher gas pressures, ${ }^{15}$ therefore $\mathrm{N}_{2}^{+}$formation is dominant. As a result, $\mathrm{N}_{2}^{+}$percentage is much higher than $\mathrm{N}^{+}$percentage in this case. When the total extracted ion beam current reaches the maximum, Fig. 3 shows that $\mathrm{N}_{2}^{+}$percentage also reaches its maximum at approximately $4.5 \times 10^{-4}$ Torr. Because gas pressure is not changed substantially, ion species ratio is minimally changed.

Figure 4 shows the ion species ratios measured in a nitrogen plasma as a function of discharge current $I_{d}$ under the following conditions: $V_{d}=120 \mathrm{~V}, V_{g}=-80 \mathrm{~V}, V_{a}$ $=-410 \mathrm{~V}$, and $p=2.0 \times 10^{-4}$ Torr. It is obvious that the $\mathrm{N}_{2}^{+}$percentage decreases and the $\mathrm{N}^{+}$percentage increases gradually with discharge current.

Increasing filament heating current (keeping gas pressure constant) results in the increase of the amount of primary electrons emitted by filaments, resulting in the increase of discharge current. As a result, discharge intensity is also increased. So, the probability of $\mathrm{N}^{+}$formation increases much faster than the probability of $\mathrm{N}_{2}^{+}$formation. Therefore, the $\mathrm{N}^{+}$percentage increases while the $\mathrm{N}_{2}^{+}$ percentage decreases with discharge current.

\section{B. Lon mass spectra in oxygen and argon plasmas}

Table I lists ion species ratios and their corresponding deflection electric field strength $E$ measured in nitrogen, oxygen, and argon plasmas under the following PSII conditions: $V_{d}=120 \mathrm{~V}, V_{g}=-80 \mathrm{~V}, V_{a}=-410 \mathrm{~V}, P=1.5$ $\times 10^{-4}$ Torr, and $I_{d}=30 \mathrm{~mA}$. 
TABLE I. Ion species ratios and corresponding deflection electric field strength $E$ in nitrogen oxygen and argon plasmas under the following conditions: $V_{d}=120 \mathrm{~V}, V_{g}=-80 \mathrm{~V}, V_{a}=-410 \mathrm{~V}, P=1.5 \times 10^{-4}$ Torr, and $Y_{d}=30 \mathrm{~mA}$.

\begin{tabular}{ccc}
\hline \hline Ion species & Ion species ratios $(\%)$ & $E(\mathrm{~V} / \mathrm{cm})$ \\
\hline $\mathrm{N}_{2}^{+}$ & 75.00 & 113.4 \\
$\mathrm{~N}^{+}$ & 25.00 & 160.4 \\
$\mathrm{O}_{2}^{+}$ & 56.66 & 106.1 \\
$\mathrm{O}^{+}$ & 43.33 & 150.0 \\
$\mathrm{Ar}^{+}$ & 83.33 & 94.9 \\
$\mathrm{Ar}^{++}$ & 16.66 & 134.2 \\
\hline
\end{tabular}

\section{ACCURACY ESTIMATION OF THE MEASURED ION SPECIES RATIO}

The ion species ratio has strong influence on the atomic composition-depth profile of the implanted target. Figure 5 shows the atomic concentration-depth profiles of the nitrogen-implanted Ti-6Al-4V target using the computer code TAMIX with different ion species ratios as the input parameter. ${ }^{16}$ It is clear that the profile varies with the changing ion species ratio. In order to estimate the accuracy of the measured ion species ratios, a flat $\mathrm{Ti}-6 \mathrm{Al}-4 \mathrm{~V}$ sample was implanted with nitrogen at a target bias of 50 $\mathrm{kV}$ and a dose of $3 \times 10^{17}$ atoms $/ \mathrm{cm}^{2}$. An experimental analysis of the atomic composition-depth profile was performed using scanning Auger microprobe (SAM).

When the ions are accelerated under the influence of the electric field inside the sheath surrounding the target, the energy distribution of the ions reaching the surface of the target depends upon the mass of the ions and the position of the ions inside the sheath. In the PSII process, the pulse applied to the target has a certain rise and fall time and the plasma contains different ions species. The range of the ions implanted into the target is a complex function of energy distribution and mass of the incident ion and the target atoms. TAMIX calculations were performed considering the energy spectrum due to PSII pulse bias and with various ion species ratios of nitrogen plasma $\left(\mathrm{N}^{+}\right.$and $\mathrm{N}_{2}^{+}$). Figure 5 shows three curves of nitrogen atomic concentration-depth profiles with different ion species ratios. In a plasma where $\mathrm{N}^{+}$ions are absent, the peak of the

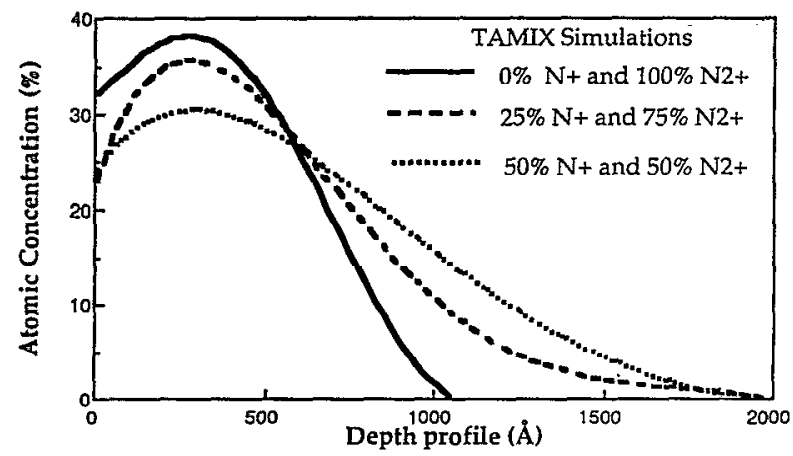

FIG. 5. Atomic concentration vs depth profile of the nitrogen implanted Ti-6AI-4V target determined from the computer code TAMIX with different ion species ratios.

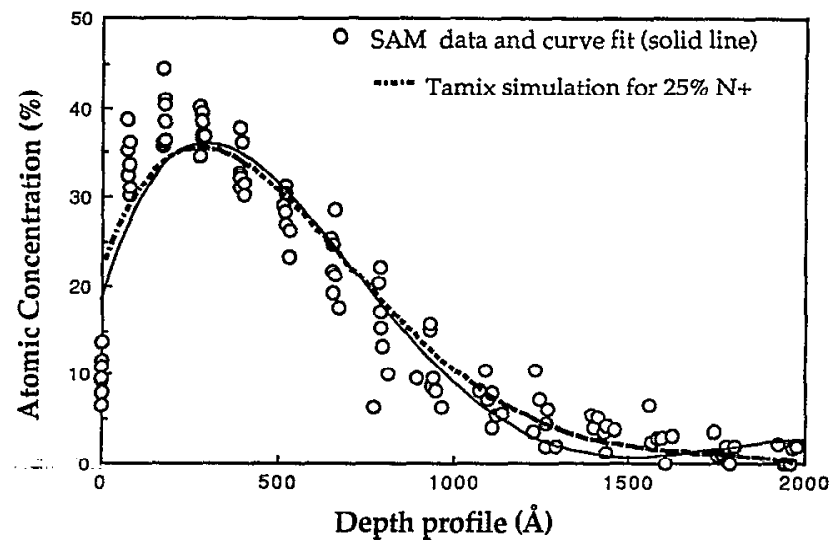

FIG. 6. Atomic concentration vs depth profile of the nitrogen implanted Ti-6Al-4V target from SAM experimental data from separate scans at different positions of the same sample and the computer code TAMIX with $25 \% \mathrm{~N}^{+}$and $75 \% \mathrm{~N}_{2}^{+}$ion species ratio.

nitrogen percentage is very high in the implanted sample but the range is small as compared to a plasma with $50 \%$ $\mathrm{N}^{+}$and $\mathrm{N}_{2}^{+}$ions. The atomic concentration-depth profile with $25 \% \mathrm{~N}^{+}$and $75 \% \mathrm{~N}_{2}^{+}$shown in Fig. 5 has been compared with SAM data in Fig. 6.

The SAM analysis for experimental determination of atomic composition-depth profile was performed at seven locations within a $1 \mathrm{~cm}^{2}$ area on the implanted sample. The atomic composition-depth profiles were generated using an Auger electron microprobe equipped with an argon ion gun at a voltage of $3 \mathrm{kV}$ to incrementally sputter atoms from the sample surface. In the present analysis, an internal calibration technique was used to overcome the problem associated with the overlap of nitrogen and titanium peaks at an energy of $385 \mathrm{eV} .{ }^{17}$ In order to convert the sputtering time to depth, for each sputtering interval, the measured composition-depth ratios were calculated by multiplying the theoretical sputter yield and the interval between compositional measurements. Finally, the depth ratios were normalized to the final depth of the sputtered crater (as measured with an alphastep profilometer). Seven SAM concentration-depth profiles were obtained from a small area $(0.7 \times 1.3 \mathrm{~mm})$ of one sample with separate scan. The dose over this small area was assumed to be uniform and the error in the repeated measurement was found to be $10 \%$ in terms of the total dose. The SAM results of nitrogen atomic concentration-depth profiles are shown in Fig. 6. A computer generated fit on the data has been plotted and a TAMIX simulation for $25 \% \mathrm{~N}^{+}$and $75 \% \mathrm{~N}_{2}^{+}$has been superimposed. The TAMIX computer simulation and Auger experimental data are in good agreement, which verifies the ions species ratio measured by the present measurement device. From the agreement, it is seen that experimental atomic composition versus depth profiles can be predicted much more accurately with the help of measurement of ion species and their ratios in PSII plasmas. 
TABLE II. The separated distance calculated from Eqs. (2) and (3) for a number of ion pairs in the radial direction at the entrance of the Faraday cup.

\begin{tabular}{lcc}
\hline \hline Ion pairs & $\begin{array}{c}\text { Separated distance } \\
(\mathrm{cm})\end{array}$ & $\begin{array}{c}\text { Deflection voltage difference } \\
(\mathrm{V})\end{array}$ \\
\hline $\mathrm{N}^{+}, \mathrm{N}^{+}$ & 3.80 & 32.88 \\
$\mathrm{O}^{+}, \mathrm{O}_{2}^{+}$ & 3.55 & 30.76 \\
$\mathrm{Ar}^{+}, \mathrm{Ar}^{++}$ & 3.18 & 27.51 \\
$\mathrm{C}^{+}, \mathrm{O}^{+}$ & 1.88 & 16.25 \\
$\mathrm{Ar}^{+}, \mathrm{ArH}^{+}$ & 0.09 & 0.81 \\
$\mathrm{CH}_{3}^{+}, \mathrm{CH}_{4}^{+}$ & 0.39 & 3.44 \\
\hline \hline
\end{tabular}

\section{DISCUSSION AND IMPROVEMENTS}

Resolution is a very important parameter of the $\mathbf{E} \times \mathbf{B}$ mass separator. Many factors influence the resolution and also give rise to peak broadening and measurement errors in the mass spectrum.

Table II lists the separated distance calculated from Eqs. (2) and (3) for a number of ion pairs in the radial direction at the entrance of the Faraday cup. A comparison between the separated distance and the diameter of the Faraday cup of $2.97 \mathrm{~mm}$ shows that the current measurement system is suitable for measurement of ion species in nitrogen, oxygen, and argon plasmas, but it can not distinguish different ion species in a methane plasma. From Eq. (4), it is obvious that an increase of deffection electric field strength $E$ and magnetic field intensity $B$ in appropriate proportion will improve the resolution substantially. Therefore, permanent magnets with stronger magnetic field intensity must be used. In order to improve the resolution, geometrical parameters $L$ and $S$ should also be increased appropriately.

Other factors can also give rise to peak broadening and measurement errors in the mass spectrum. $\Lambda$ s mentioned above, if a lower gas pressure is maintained inside the measurement system, extracted ion beam scattering will be reduced, resulting in improvement of peak broadening. As a consequence, an independent pumping system for the present measurement system would be desirable. In general, an extracted ion beam is more or less divergent. Ion beam divergence results in peak broadening. A focusing lens or collimator can improve the peak broadening problem substantially. Manufacturing and alignment errors, dispersion of the deflection magnetic field, instability of power supply can also result in peak broadening and measurement errors and should be improved as much as possible.

From the agreement between the TAMIX computer simulation and SAM experimental data and the analysis and discussion mentioned above, it is enough to prove that this kind of the measurement system can be used in principle for measuring ion species ratios in the PSII device. An independent pumping system and permanent magnets with stronger magnetic field intensity will ensure higher resolution to measure ion species in all PSII plasmas.

\section{ACKNOWLEDGMENTS}

The authors would like to acknowledge Sridharan $\mathrm{Ku}-$ mar and Doyle Patti for their technical assistance. This work was supported by NSF Grant Nos. DMC-8712461 and UMSI rescarch proposal Grant No. MG 32804.

'J. R. Conrad, J. Appl. Phys. 62, 777 (1987).

${ }^{2}$ R. Conrad, R. A. Dodd, F. J. Worzala, and X. Qiu, Surf. Coatings Technol. 36, 927 (1988).

${ }^{3}$ J. T. Scheuer, M. Shamim, and J. R. Conrad, J. Appl. Phys. 67, 1241 (1990).

${ }^{4}$ J. R. Conrad, J. L. Radtke, R. A. Dodd, and F. J. Worzala, J. Appl. Phys. 62, 4591 (1987).

${ }^{5}$ A. Chen, J. T. Scheuer, C. Ritter, R. B. Alexander, and J. R. Conrad, J. Appl. Phys. 70, 6757 (1991).

${ }^{6}$ J. Tendys and I. J. Donnelly, Appl. Phys. Lett. 53, 2143 (1988).

${ }^{7}$ J. W. Coburn, Rev. Sci. Instrum. 41, 1219 (1970).

${ }^{8}$ E. Y. Wang, L. Schmitz, Y. Ra, B. LaBombard, and R. W. Conn, Rev. Sci. Instrum. 61, 2155 (1990).

${ }^{9}$ L. Bartha, A. Z. Kiss, E. Koltay, and Gy. Szabo, Nucl. Instrum. Methods A 287, 156 (1990).

${ }^{10}$ S. Tanaka, M. Akiba, M. Araki, S. Matsuda, Y. Matsuda, Y. Ohara, Y. Okumura, K. Yokoyama, and K. Watanabe, Nucl. Instrum Methods B 37, 128 (1989).

${ }^{11}$ M. R. Shubaly, R. G. Maggs, and A. E. Weeden, IEEE Trans. Nucl. Sci. NS-32, 1751 (1985).

${ }^{12}$ G. Aston, H. R. Kaufman, and P. J. Wilbur, AIAA J. 16, 516 (1978).

${ }^{13}$ L. Wahlin, Nucl. Instrum. Methods 27, 55 (1964).

${ }^{14}$ K. Jensen and E. Veje, Nucl. Instrum. Methods 122, 511 (1974).

${ }^{15}$ S. N. Misra and S. K. Gupta, Nucl. Instrum. Methods 122, 303 (1974).

${ }^{16}$ S. H. Han, G. L. Kulcinski, and J. R. Conrad, Nucl. Instrum. Methods B 45, 701 (1990).

${ }^{17}$ P. T. Dawson and K. K. Tzatzov, Surf. Sci. 149, 105 (1985). 